\title{
Lorentz violation and higher-derivative gravity
}

\author{
C. A. Hernaski* \\ Indiana University Center for Spacetime Symmetries, Bloomington, Indiana 47405, USA \\ H. Belich ${ }^{\dagger}$ \\ Departamento de Física e Química, \\ Universidade Federal do Espírito Santo, Avenida Fernando Ferrari, \\ 514, Goiabeiras, 29060-900, Vitória, ES, Brazil.
}

\begin{abstract}
In this work, we analyze a gravity model with higher derivatives including a CPT-even Lorentzviolating term. In principle, the model could be a low-energy limit of a Lorentz-invariant theory presenting the violation of Lorentz symmetry as a consequence of a spontaneous symmetry-breaking mechanism if a decoupling between the metric and the Nambu-Goldstone modes is assumed. We have set up a convenient operator basis for the expansion of wave operators for symmetric secondrank tensors in the presence of a background vector. By using this set of operators, the particle content is obtained, and its consistency, regarding the conditions for stability and unitarity, is discussed. We conclude that this extra Lorentz noninvariant contribution is unable to address the problems of stability and unitarity of higher-derivative gravity models.
\end{abstract}

PACS numbers: 04.60.-m, 04.50.Kd, 11.30.Er

Keywords: Lorentz Breaking, Gravity Models, Higher Derivatives

*Electronic address: chernask@indiana.edu

†Electronic address: belichjr@gmail.com 


\section{INTRODUCTION}

Problems involving the consistent quantization of the gravitational interaction have been widely discussed and are well known. The standard quantization of the Einstein-Hilbert (EH) Lagrangian using the formalism of perturbative quantum field theory (QFT) turns out to be inadequate, since it results in a non-renormalizable model [1]. Attempts to formulate a consistent quantum gravity model in four spacetime dimensions led to the proposal of additional terms involving higher derivatives. These terms modify the propagating structure of the excitations (including the graviton) in order to improve the ultraviolet (UV) behavior of the scattering amplitudes [2-4]. However, invariably, they also introduce negative-norm excitations called ghost particles that damage the unitarity of the $\mathrm{S}$ matrix. As discussed

in Ref. [5], this is a general feature of gravitational models built up with diffeomorphismcovariant terms and points to an incompatibility between renormalizability and unitarity of the S matrix.

[2 6]. An interesting possibility that evades some previous assumptions is to consider the Lorentz and diffeomorphism-symmetry-breaking effects on these gravity models, which, in turn, should describe the phenomenological aspects of general relativity (GR) in a low-energy limit.

The interest in models that present the breaking of the Lorentz symmetry has increased after Kostelecký and Samuel showed that string theory, one of the leading candidates to handle the issue of consistent quantization of gravity, may present some phases where Lorentz symmetry is violated through a spontaneous symmetry-breaking mechanism triggered by the appearance of nonvanishing expectation vacuum values of nontrivial Lorentz tensors [7]. In this case, the models with Lorentz symmetry breaking are considered effective, and the analysis of their phenomenological aspects at low energies may provide information and impose restrictions on the fundamental theory from which they stem.

A general framework for testing the low-energy manifestations of CPT and Lorentz symmetry breaking is the Standard-Model Extension (SME) [8]. In this framework, the effective Lagrangian corresponds to the usual Lagrangian of the Standard Model (SM), to which is added SM operators of any dimensionality contracted with Lorentz-violating (LV) tensorial background coefficients. The effective Lagrangian is written in a Lorentz-invariant form 
under coordinate transformations to guarantee the observer independence of physics. However, the physically relevant transformations are those that affect only the dynamical fields of the theory. These changes are called particle transformations, whereas the coordinate transformations (including the background tensor) are called observer transformations. In Refs. [9, 10], these concepts are more deeply analyzed.

To contemplate the possible consequences of CPT and LV for experiments where the gravitational interaction plays an important role, gravity was also included in the general framework of the SME [11]. One of the most striking consequences of this fusion is the requirement of a dynamic mechanism for the diffeomorphism/Lorentz-invariance violation - that is, the violation of these spacetime symmetries should be spontaneous to be consistent with the geometrical aspects of the gravitational interaction [12]. In practice, this means that the tensor coefficients appearing in the diffeomorphism/Lorentz-violating operators must be replaced by dynamical fields, and these, in turn, under a specific dynamic mechanism, acquire nonvanishing vacuum expectation values. As compared with the scenario of explicit breaking, one finds extra Nambu-Goldstone and massive modes that are responsible for restoring the particle diffeomorphism/Lorentz invariance of the model [13, 14].

Concerning the experimental searches for the CPT/Lorentz-violation signals, the generality of the SME has provided the basis for many investigations. In the flat spacetime limit, the empirical studies include muons [15], mesons [16, 17], baryons [18], photons [19], electrons [20], neutrinos [21], and the Higgs [22] sector. The gravity sector was also explored in Refs. [23 26]. In Ref. [27], one can find the current limits on coefficients for Lorentz violation.

The aim of this work is to investigate if, by considering a diffeomorphism/Lorentzviolating CPT-even term, one can get a model with the spectrum free from instabilities and, at the same time, accompanied by higher derivatives, since an improved UV behavior of the scattering amplitudes is expected in the presence of such terms. For the discussion of these issues, we have analyzed the properties of the particle propagators that show up in this model. For this task, we have concentrated on the quadratic Lagrangian resulting from the expansion of the dynamical fields around a vacuum solution to the equations of motion. For simplicity, we have decided to focus only on the propagating modes that come from the dynamical metric, leaving aside the Nambu-Goldstone and massive modes that should appear as the result of the spontaneous symmetry-breaking mechanism. This choice 
relies on the fact that one can write interacting Lagrangians where these extra modes decouple from the metric modes, and one can study the properties of the latter independently of the former. However, one should be aware that the full consistency of the interacting system should include these extra modes. We hope that this inconsistency does not appear perturbatively at the quadratic level, but this possibility should be explicitly investigated [28]. Assuming the validity of such a decoupling, we work out the propagators of the model using a suitable operator basis for the wave operator that highlights the physical degrees of freedom (d.o.f.) propagating in the model, as well as keeping track of the role of the Lorentz breaking in providing extra couplings between these d.o.f. In the following, we perform a general analysis of the stability and unitarity, pointing out the extra subtleties brought by the LV. Our conclusion is that the extra room obtained by removing the Lorentz invariance is not enough to render the higher-derivative model consistent. Unless we consider the model from a effective point of view and assume some parameters of the Lagrangian to be of the same order as some assumed cutoff, the model has insurmountable consistency difficulties even in the infrared regime.

This paper is organized as follows: In Sec. III, we set up the notation and conventions to be used, and we start with a general Lagrangian containing the above mentioned LV term in addition to the EH and curvature-square terms. The collection and analysis of propagators is carried out in Sec. IIA. The conditions for stability of the spectrum are attained in Sec. IIB. In Sec. III, we present our discussions and conclusions.

\section{GRAVITATION WITH HIGHER DERIVATIVES AND VIOLATION OF LORENTZ SYMMETRY}

In Ref. [29] is carried out an analysis of the spectral consistency of a gravitational model in $3+1$ dimensions that contains, in addition to the EH term, terms with higher derivatives $\left(R^{2}\right.$ and $\left.R_{a b} R^{a b}\right)$ plus two CPT-odd terms which manifest the breaking of the CPT and diffeomorphism/Lorentz symmetry through a constant background vector: a ChernSimons-like and a Ricci-Cotton-like term. As in the case of gravitation without diffeomorphism/Lorentz breaking, we concluded that the higher-derivative terms should be avoided to get a stable model. The only consistent combination is the EH Lagrangian added to the Chern-Simons-type term with a timelike background vector. In this work, we address the 
analysis of the compatibility of unitarity with higher-derivative terms by considering a CPTeven diffeomorphism/Lorentz-violating operator. We consider the following Lagrangian:

$$
\mathcal{L}=\sqrt{-g}\left(\alpha R+\beta R_{a b} R^{a b}+\gamma R^{2}+\frac{1}{4} \kappa^{a b c d} R_{a b f g} R^{f g}{ }_{c d}\right) .
$$

The parameters $\alpha, \beta$, and $\gamma$ are arbitrary, and the background tensor $\kappa^{a b c d}$ is responsible for the breakdown of the diffeomorphism invariance that, whenever we consider the linearized model, will lead to the breaking of Lorentz symmetry. To simplify the discussion, we will make the additional assumption that this tensor is constructed with a background vector $b^{a}$ [7]. Then, we have

$$
\kappa^{a b c d}=\frac{1}{2}\left(\kappa^{a c} g^{b d}+\kappa^{b d} g^{a c}-\kappa^{b c} g^{a d}-\kappa^{a d} g^{b c}\right),
$$

with

$$
\kappa^{a b}=\kappa\left(b^{a} b^{b}-\frac{1}{4} g^{a b} b \cdot b\right)
$$

and

$$
\kappa=\frac{4}{3} \kappa^{a b} b_{a} b_{b} .
$$

The conventions adopted for the Riemann tensor, Ricci and scalar curvature are those followed by Ref. [32]. That is,

$$
\begin{aligned}
R_{b c d}^{a} & =\partial_{c} \Gamma_{b d}^{a}-\partial_{d} \Gamma_{b c}^{a}+\Gamma_{c e}^{a} \Gamma_{b d}^{e}-\Gamma_{d e}^{a} \Gamma_{b c}^{e}, \\
R_{b c} & =R_{b a c}^{a}, \quad R=g^{a b} R_{a b},
\end{aligned}
$$

and the Christoffel symbol $\Gamma_{b c}^{a}$ is given by

$$
\Gamma_{b c}^{a}=\frac{1}{2} g^{a d}\left(\partial_{b} g_{d c}+\partial_{c} g_{d b}-\partial_{d} g_{b c}\right) .
$$

The background tensor given in Eq. (22) has the same symmetries as the Riemann tensor, as can easily be seen by the structure of the breaking term in Eq. (11). We can assume that the origin of the background vector, $b^{a}$, is due to a spontaneous breaking of Lorentz symmetry in a more fundamental theory, as for example in string theory [7]. The extra modes coming from the spontaneous symmetry-breaking mechanism are not discussed, and we expect that for some classes of models this can be done independently without disturbing the present analysis, as discussed in the Introduction. Furthermore, the nature of the background vector, if it is space-, time-, or lightlike, is not assumed a priori. We discuss the consistency of the model, case by case, in the following sections. The values of the constants appearing in Eq. 
(1) must be prescribed in such a way to obtain a model with a particle content free of ghosts and tachyons.

The Minkowski metric corresponds to a possible solution of the Euler-Lagrange equations obtained from Eq. (11). Therefore, we can consider a perturbation of the metric field around the Minkowski metric,

$$
g_{a b}=\eta_{a b}+h_{a b},
$$

where $\eta_{a b}=\operatorname{diag}(1,-1,-1,-1)$. In terms of $h_{a b}$, the Lagrangian [Eq. (11)] establishes the dynamics for this field in an anisotropic spacetime scenario. Furthermore, we will assume that the background vector is constant in the asymptotic Minkowski coordinates

$$
\partial_{a} b^{c}=0
$$

We should highlight that this condition is not equivalent to the covariant constancy of the background vector, $b_{\mu}$, under general diffeomorphisms. The (covariant) constancy of $b_{\mu}$ does not hold for arbitrary manifolds; it rather imposes a constraint on the curved space [11]. For that reason, we are not assuming $b_{\mu}$ to be covariantly constant. We relax this more stringent condition and, in simply considering metric fluctuations (weak-field approximation), it is legitimate to consider constancy in the asymptotic Minkowskian sense, as stated above in Eq. (9).

The particle content described by this model can be examined considering the propagators, whose structures depend only on the quadratic Lagrangian in $h_{a b}$. Up to total derivatives, it is given by

$$
\begin{aligned}
(\mathcal{L})_{2} & =\frac{\alpha}{2}\left(\frac{1}{2} h^{\mu \nu} \square h_{\mu \nu}-\frac{1}{2} h \square h+h \partial_{\mu} \partial_{\nu} h^{\mu \nu}-h^{\mu \nu} \partial_{\mu} \partial_{\lambda} h^{\lambda}{ }_{\nu}\right) \\
& +\frac{\beta}{2}\left(h \square^{2} h-2 h \square \partial_{\mu} \partial_{\nu} h^{\mu \nu}+h^{\mu \nu} \partial_{\mu} \partial_{\nu} \partial_{\kappa} \partial_{\lambda} h^{\kappa \lambda}\right) \\
& +\frac{\gamma}{8}\left(h^{\mu \nu} \square^{2} h_{\mu \nu}-2 h \square \partial_{\mu} \partial_{\nu} h^{\mu \nu}+2 h^{\mu \nu} \partial_{\mu} \partial_{\nu} \partial_{\kappa} \partial_{\lambda} h^{\kappa \lambda}+h \square^{2} h\right) \\
& +\frac{\kappa b^{a} b^{c}}{4}\left(2 h_{a g} \partial_{f} \partial^{d} \partial_{c} \partial^{g} h_{d}^{f}-h_{g}^{d} \partial_{f} \partial_{a} \partial_{c} \partial^{g} h_{d}^{f}\right. \\
& \left.-2 h_{a f} \partial^{d} \partial_{c} \square h_{d}^{f}{ }_{d}+h^{d}{ }_{f} \partial_{a} \partial_{c} \square h^{f}{ }_{d}-h_{a g} \partial_{f} \partial^{g} \square h^{f}{ }_{c}+h_{a f} \square^{2} h^{f}{ }_{c}\right) \\
& -\frac{\kappa b \cdot b}{8}\left(h_{a g} \partial_{f} \partial^{d} \partial^{a} \partial^{g} h_{d}^{f}+h_{f}^{d} \square^{2} h^{f}{ }_{d}-2 h_{a f} \partial^{d} \partial^{a} \square h^{f}{ }_{d}\right) .
\end{aligned}
$$

It is important to notice that, although the background vector breaks the diffeomorphism invariance of the Lagrangian [Eq. (1)], the quadratic Lagrangian has the gauge invariance

$$
h_{a b}^{\prime}=h_{a b}+\partial_{a} \xi_{b}+\partial_{a} \xi_{b} .
$$


This is due to the fact that the model in Eq. (1) is constructed using only scalar and tensor curvatures. Under general diffeomorphism transformations, these quantities transform covariantly (or invariantly in the case of scalars). When considering the linear contribution in the field $h_{a b}$, these terms are invariant under the gauge transformation [Eq. (11)] arising from the linearized diffeomorphisms

$$
x^{\prime \mu}=x^{\mu}+\xi^{\mu}(x) .
$$

As in GR, the gauge invariance in Eq. (11) is responsible for inhibiting the propagation of a spin-1 and a spin-0 mode [30]. Moreover, dynamically, the graviton keeps its two helicity d.o.f, as we shall see in the next section.

\section{A. Obtaining the propagators}

To characterize the particle content that propagates in this spacetime scenario, we analyze the propagator structure of the excitations. Then, we first write Eq. (10) as follows:

$$
(\mathcal{L})_{2}=\frac{1}{2} h^{a b} \mathcal{O}_{a b ; c d} h^{c d}
$$

where $\mathcal{O}$ is the wave operator. The task of obtaining the propagators is equivalent to finding the inverse of this operator. For this, we will use an algebraic method by finding an operator basis in terms of which we can expand it. The two main criteria for the construction of this basis will be algebraic simplicity and easy physical interpretation. As we will see later, the

first criterion is fulfilled if we build an operator basis consisting of projectors and mappers. The vector space of the field in question is then divided into subspaces defined by projectors, and those with the same dimension can be mapped in each other by means of the mappers. Our interest is in the particle content described by the model. Thus, in order to satisfy the second criterion, we define the subspaces in such a way as to associate a well-defined spin to the d.o.f. of the fields that reside in these subspaces.

In models with Lorentz invariance, the kinetic terms of the Lagrangian are built using only metrics and derivatives. Thus, the kinetic term for the field $h^{a b}$, if it is Lorentz invariant, should be such that the wave operator could be expanded in terms of Barnes-Rivers 
operators [31]. These, in turn, are given by

$$
\begin{aligned}
P(2)_{a b ; c d} & \left.=\frac{1}{2}\left(\theta_{a c} \theta_{b d}+\theta_{a d} \theta_{b c}\right)-\frac{1}{3} \theta_{a b} \theta_{c d}\right), \\
P(1)_{a b ; c d} & =\frac{1}{2}\left(\theta_{a c} \omega_{b d}+\theta_{a d} \omega_{b c}+\theta_{b c} \omega_{a d}+\theta_{b d} \omega_{a c}\right), \\
P_{11}(0)_{a b ; c d} & =\frac{1}{3} \theta_{a b} \theta_{c d}, \\
P_{22}(0)_{a b ; c d} & =\omega_{a b} \omega_{c d}, \\
P_{12}(0)_{a b ; c d} & =\frac{1}{\sqrt{3}} \theta_{a b} \omega_{c d}, \\
P_{21}(0)_{a b ; c d} & =\frac{1}{\sqrt{3}} \omega_{a b} \theta_{c d} .
\end{aligned}
$$

However, the presence of background tensors allows extra couplings to the fields in such a way that the Barnes-Rivers operators are, in general, insufficient to expand the wave operators containing these structures.

We can arrange the terms of the quadratic Lagrangian [Eq. (10)] into two distinct classes. One is composed of terms in which at least one of the $h$ fields is contracted with the background vector, and the other presents terms in which the background vectors are contracted with each other or with the derivatives. Thus, we rewrite Eq. (13) as follows:

$$
(\mathcal{L})_{2}=\frac{1}{2} h^{a b} \mathcal{O}_{a b ; c d}^{1} h^{c d}+\frac{1}{2} h^{a b} \mathcal{O}_{a b ; c d}^{2} h^{c d},
$$

with

$$
\begin{aligned}
\mathcal{O}_{a b ; c d}^{1}= & \frac{1}{2} \kappa p^{2}\left[-(v \cdot p)\left(v_{d} \eta_{b c} p_{a}-v_{a} \omega_{c d} p_{b}+v_{a} \eta_{b c} p_{d}\right.\right. \\
& \left.\left.-v_{d} \omega_{a b} p_{c}\right)+p^{2}\left(v_{a} v_{d} \eta_{b c}-v_{a} v_{d} \omega_{b c}\right)\right]+\cdots
\end{aligned}
$$

and

$$
\begin{aligned}
\mathcal{O}^{2} & =\alpha p^{2}\left(\frac{1}{2} \eta_{a c} \eta_{b d}-\frac{1}{2} \eta_{a b} \eta_{c d}-\eta_{a c} \omega_{b d}+\eta_{a b} \omega_{c d}\right) \\
& +\beta p^{4}\left(\frac{1}{2} \eta_{a c} \eta_{b d}+\frac{11}{6} \eta_{a b} \eta_{c d}+\frac{7}{3} \omega_{a b} \omega_{c d}-\eta_{a c} \omega_{b d}-\frac{11}{3} \eta_{a b} \omega_{c d}\right) \\
& +\frac{2}{3} \gamma p^{4}\left(\eta_{a b} \eta_{c d}-2 \eta_{a b} \omega_{c d}+\omega_{a b} \omega_{c d}\right) \\
& -\frac{1}{4} \kappa v^{2} p^{4}\left(\eta_{a c} \eta_{b d}+\frac{13}{3} \omega_{a b} \omega_{c d}-2 \eta_{a c} \omega_{b d}+\frac{10}{3} \eta_{a b} \eta_{c d}-\frac{20}{3} \eta_{a b} \omega_{c d}\right) \\
& +\frac{1}{2} \kappa p^{2}(v \cdot p)^{2}\left(\eta_{b c} \eta_{a d}-\eta_{a d} \omega_{b c}\right)+\cdots .
\end{aligned}
$$


The ellipses in the above expressions refer to terms with the same prior structure, but switching $a \longleftrightarrow b, b \leftrightarrow c, a b \leftrightarrow c d$ in such a way that the wave operator is symmetric under these exchanges.

The fundamental structures that effectively contribute to the operational character of $\mathcal{O}^{2}$ correspond to metrics and derivatives or metrics and momenta in Fourier space. Thus, we expect to expand $\mathcal{O}^{2}$ in terms of the Barnes-Rivers operators [Eqs. (14) - (19)]. In fact, one can show that

$$
\begin{aligned}
\mathcal{O}_{a b ; c d}^{2} & =\frac{1}{2} p^{2}\left\{\left(\alpha+\left(\beta-\frac{1}{2} \kappa b^{2}\right) p^{2}\right) P(2)+\left[\left(6 \beta+2 \gamma-\frac{11}{4} \kappa b^{2}\right) p^{2}-\alpha\right] P_{11}(0)\right\}_{a b ; c d} \\
& +\frac{1}{2} \kappa p^{2}(b \cdot p)^{2}\left(P(2)+P_{11}(0)+\frac{1}{2} P(1)\right)_{a b ; c d} .
\end{aligned}
$$

Once the $\mathcal{O}^{1}$ operator contains in its internal structure the background vector, $b_{a}$, we cannot expand it in terms of the Barnes-Rivers operators. The direction in spacetime defined by $b_{a}$ breaks Lorentz symmetry, promoting the coupling between the d.o.f. of the distinct spins defined by Eq. (14) - (17). In the cases of spins 1 and 2, which define subspaces with dimension 3 and 5, respectively, the background vector yields a complete splitting into onedimensional subspaces. Furthermore, the structure of the terms in Eq. (21) establishes the possible coupling between these d.o.f. In order to present the projectors that compose the Barnes-Rivers operators, we firstly write the background vector in terms of the momentum and of a spacelike vector as

$$
b_{a}=\frac{b \cdot p}{p^{2}} p_{a}+\sqrt{\frac{p_{*}^{2}}{p^{2}}} e_{a}^{3},
$$

where

$$
p_{*}^{2}=(b \cdot p)^{2}-b^{2} p^{2}
$$

and $e_{a}^{3}$ is a spacelike vector orthogonal to $p$. If we define $e_{1}$ and $e_{2}$ such that

$$
e_{i} \cdot e_{j}=-\delta_{i j}, \quad e_{i} \cdot p=0
$$

we can split the transverse operator as

$$
\theta=\rho+\sigma+\tau
$$

with

$$
\rho_{a b}=-e_{a}^{1} e_{b}^{1}, \quad \sigma_{a b}=-e_{a}^{2} e_{b}^{2}, \quad \tau_{a b}=-e_{a}^{3} e_{b}^{3} .
$$


With these definitions, we can split the Barnes-Rivers operators in terms of projectors which act in each one of the spin subspaces. Also, we can define mappers among the subspaces defined by these projectors. Any operator, projector, or mapper can be written, up to changes of basis by unitary transformations, as

$$
P(I J)_{(i j)}=(-)^{R+S} \psi(I)_{(i)} \psi(J)_{(j)},
$$

where $\psi(I)_{(i)}$ is the $i$ th eigenvector of the Barnes-Rivers spin projector $P(I)$. $R, S$ can assume the values 0 and 1 , corresponding to the spin parities +1 or -1 , respectively. By convention, we choose the subscripts $i$ and $j$ to range from 1 to 5 for the spin $2(I, J=2)$, to assume the values 6 and 7 for the 0-spins $(I, J=0)$ defined by Eqs. (16) and (17), respectively, and to range from 8 to 10 for the spin $1(I, J=1)$. In terms of the vectors in Eq. (26), we can write the $\psi$ eigenvectors as

$$
\begin{aligned}
& \psi(2)_{(1) a b}=\frac{1}{\sqrt{2}}\left(e_{1 a} e_{2 b}+e_{2 a} e_{1 b}\right) \\
& \psi(2)_{(2) a b}=\frac{1}{\sqrt{2}}\left(e_{1 a} e_{3 b}+e_{3 a} e_{1 b}\right) \\
& \psi(2)_{(3) a b}=\frac{1}{\sqrt{2}}\left(e_{2 a} e_{3 b}+e_{3 a} e_{2 b}\right) \\
& \psi(2)_{(4) a b}=\frac{1}{\sqrt{2}}\left(\rho_{a b}-\sigma_{a b}\right) \\
& \psi(2)_{(5) a b}=\frac{1}{\sqrt{6}}\left(\rho_{a b}+\sigma_{a b}-2 \tau_{a b}\right) \\
& \psi(0)_{(6) a b}=\frac{1}{\sqrt{3}} \theta_{a b}, \\
& \psi(0)_{(7) a b}=\omega_{a b}, \\
& \psi(1)_{(8) a b}=-\frac{1}{\sqrt{2}}\left(e_{a}^{1} \frac{p_{b}}{\sqrt{p^{2}}}+e_{b}^{1} \frac{p_{a}}{\sqrt{p^{2}}}\right) \\
& \psi(1)_{(9) a b}=-\frac{1}{\sqrt{2}}\left(e_{a}^{2} \frac{p_{b}}{\sqrt{p^{2}}}+e_{b}^{2} \frac{p_{a}}{\sqrt{p^{2}}}\right) \\
& \psi(1)_{(10) a b}=-\frac{1}{\sqrt{2}}\left(e_{a}^{3} \frac{p_{b}}{\sqrt{p^{2}}}+e_{b}^{3} \frac{p_{a}}{\sqrt{p^{2}}}\right) .
\end{aligned}
$$

Using Eq. (26), the normalization of the $\psi$ eigenvectors is given by

$$
\psi(I)_{(i) a b} \psi(J)_{(j)}^{a b}=(-)^{P} \delta^{I J} \delta_{i j}
$$

The operators of the type $P_{i j}(J M)$ are objects that map the subspace defined by $P_{i i}(J J)$ 
in the subspace defined by $P_{j j}(M M)$. The algebra that these operators satisfy is orthonormal and complete in the following sense:

$$
\begin{aligned}
P_{i j}(J M)_{a b ; f g} P_{k l}(N P)_{c d}^{f g} & =\delta_{j k} \delta_{M N} P_{i l}(J P)_{a b ; c d} \\
\sum_{i, J} P_{i i}(J J) & =1 .
\end{aligned}
$$

In terms of these operators, we can write $\mathcal{O}^{1}$ as follows:

$$
\begin{aligned}
\mathcal{O}^{1} & =\frac{1}{2} \kappa p^{2}\left[\frac{1}{2}\left((b \cdot p)^{2}+b^{2} p^{2}\right)\left(P_{22}(2-2)+P_{33}(2-2)\right)\right. \\
& +\frac{1}{3}\left((b \cdot p)^{2}+2 b^{2} p^{2}\right) P_{55}(2-2)+(b \cdot p)^{2}\left(P_{11}(2-2)\right. \\
& \left.+P_{44}(2-2)\right)+\frac{1}{3}\left(2(b \cdot p)^{2}+b^{2} p^{2}\right) P_{11}(0-0) \\
& \left.+\frac{\sqrt{2}}{3}\left((b \cdot p)^{2}-b^{2} p^{2}\right)\left(P_{15}(0-2)+P_{51}(2-0)\right)\right] .
\end{aligned}
$$

The expansion of the wave operator in terms of the operators in Eq. (41) presents, in general, coefficients $a(J M)$ organized in matrix blocks that show a coupling between the $J$ and $M$ spins. To avoid redundancy in the notation, we denote the coefficient matrices associated with operators of the $J$ and $M$ spins simply as $a(J M)$, with a random order in the appearance of the $J$ and $M$ letters. Thus, the $a(J J), a(M M)$, and $a(M J)$ coefficients are parts of the same matrix $a(J M)$ in such a way that a wave operator is generally written as

$$
\mathcal{O}=\sum_{i j} a_{i j}(J M) P_{i j}(J M)
$$

The sum over the $J M$ indices is already contained in the sum over the $i j$ ones, according to our notation in Eqs. (30) - (39).

In the case where the matrices $a(J M)$ are invertible, the propagator saturated with the emission and absorption sources of particles is given by

$$
\Pi=i \sum_{i j} a^{-1}(J M)_{i j} J^{* a b} P_{i j}(J M)_{a b ; c d} J^{c d}
$$

However, as already discussed, the Lagrangian of Eq. (10) has the gauge symmetry of Eq. (11), which implies the absence of spin-1 and spin-0 sectors, as defined by Eqs. (15) and (17), respectively. In this case, the wave operator is not invertible. Nevertheless, the gauge invariance also requires that the sources of emission and absorption satisfy conditions such 
that there is no emission and absorption of the modes that depend on the gauge. Thus, the contractions of the spin-1 and spin-0 operators [Eqs. (15) and (17)] with the sources should be null, resulting in the gauge invariant propagator given by Eq. (45) with only the inverses of the largest nondegenerate submatrices.

In our model, the complete wave operator [Eq. (44)] presents only a nonvanishing matrix, that is given by

$$
a(02)=\left(\begin{array}{cccccc}
A & 0 & 0 & 0 & 0 & 0 \\
0 & B & 0 & 0 & 0 & 0 \\
0 & 0 & B & 0 & 0 & 0 \\
0 & 0 & 0 & A & 0 & 0 \\
0 & 0 & 0 & 0 & C & F \\
0 & 0 & 0 & 0 & F & D
\end{array}\right),
$$

with

$$
\begin{aligned}
& A=\frac{1}{2} p^{2}\left(\alpha+\beta p^{2}+\kappa\left((b \cdot p)^{2}-\frac{1}{2} b^{2} p^{2}\right)\right) \\
& B=\frac{1}{2} p^{2}\left(\alpha+\beta p^{2}+\frac{1}{2} \kappa(b \cdot p)^{2}\right) \\
& C=\frac{1}{2} p^{2}\left(\alpha+\beta p^{2}+\frac{1}{3} \kappa\left((b \cdot p)^{2}+\frac{1}{2} b^{2} p^{2}\right)\right), \\
& D=p^{2}\left[(6 \beta+2 \gamma) p^{2}-\alpha\right]+\frac{1}{6} \kappa p^{2}\left(2(b \cdot p)^{2}-\frac{31}{2} b^{2} p^{2}\right), \\
& F=\frac{\sqrt{2}}{6} \kappa p^{2}\left((b \cdot p)^{2}-b^{2} p^{2}\right) .
\end{aligned}
$$

The constraints satisfied by the sources are given by

$$
\begin{gathered}
p_{a} \mathcal{J}^{a b}=p_{a} \mathcal{J}^{b a}=0 \\
\tau_{a b} \mathcal{J}^{b c}=\tau_{a b} \mathcal{J}^{c b}=0
\end{gathered}
$$

where the last condition is valid only on the mass shell. These identities are responsible for the inhibition of the spin-1 and spin-0 modes associated with the gauge invariance of the model.

As we can see, the matrix $a(02)$ assumes a block-diagonal form. The lower-right block points to a coupling between the spin 0 and the fifth component of the spin 2 . The other components of the spin 2 do not couple to the other spins. However, the distinction of the coefficients, $A$ and $B$, indicates that the propagation of components 1 and 4 have dynamics 
independent of the components 2 and 3. This splitting of the spin-2 matrix into direct-sumof- $U$ (1) complex-conjugate-related representations is compatible with the CPT invariance and the breaking of the Lorentz symmetry by a background vector. The structure of the $a(02)$ matrix suggests that we can split it into three distinct blocks:

$$
\begin{aligned}
& a^{14}(2)=\left(\begin{array}{ll}
A & 0 \\
0 & A
\end{array}\right), \\
& a^{23}(2)=\left(\begin{array}{ll}
B & 0 \\
0 & B
\end{array}\right), \\
& a^{56}(02)=\left(\begin{array}{ll}
C & F \\
F & D
\end{array}\right) .
\end{aligned}
$$

The matrices $a^{14}$ and $a^{23}$ only carry information about the dynamics of the spin 2 , while the matrix $a^{56}$ shows the conjunction of the spin-0 d.o.f. with one of the d.o.f. of the spin 2 .

\section{B. Analysis of the particle content}

To analyze whether the particle content described by the model in Eq. (20) is tachyon and ghost free, we explore the structure of the propagator [Eq. (45)] expressed in terms of the inverses of the matrices in Eqs. (54) - (566). The saturated propagator has poles when the determinants of these matrices vanish. In a Lorentz-invariant model, the zeros of these determinants always occur for values of $p^{2}$ such that $p^{2}=m^{2}$, with $m^{2}$ being defined as the particle mass. The condition for the absence of tachyons in this case is given by $m^{2}>0$. This condition assures positive-energy modes for any value of the momentum $\vec{p}$, preventing the arising of instabilities due to the absence of a minimum energy.

For LV, the poles of the propagators may take forms more general than $p^{2}=m^{2}$, due to the presence of the background tensors. In this case, the discussion of stability is a much more subtle issue. The positivity of energy for all $\vec{p}$ and the observer Lorentz invariance are insufficient to prevent the appearance of spacelike momenta in the dispersion relations. In a strongly boosted frame, this implies the reemergence of negative energies, spoiling the supposed stability of the model. So, besides requiring the positivity of the energy for all $\vec{p}$ in one frame, one must also impose that spacelike momenta are absent or, equivalently, that the energy is positive in all frames. 
For a general quadratic form built from the energy and momentum, these requirements imply that the particle and antiparticle energy solutions of the dispersion relations should correspond to positive and negative branches of a hyperbola whose asymptotes lie inside or on the light cone in the Minkowski causal diagram.

In spite of the possible solution to the stability problem in the way we have described, this pattern of dispersion relation raises other problems related to microcausality [33 36]. In fact, as discussed in Ref. [33], the microcausality condition, that expresses that fields should commute for spacelike separations of their arguments, imposes that the modulus of the group velocity of the wave packets, formed out from the superposition of plane waves that satisfy the associated dispersion relations, must be lower than or at most equal to 1 (in natural units). But this requirement, in general, clashes with the stability constraint, since there, the slopes of the asymptotes are the limit of the group velocity and, as discussed above, should be greater than or at least equal to 1. Apparently, the only exception is the limiting situation where the asymptotes lie exactly on the light cone.

The mentioned difficulty in simultaneously attaining stability and causality in LV models originates in the role that these assumptions play in the search for a Lorentz-invariant description of quantum theory. For the moment, we must only discuss the conditions of stability and unitarity and shall comment where the microcausal problem can appear. A detailed investigation of this question should be considered, taking into account the role of the extra modes arising from the SSB mechanism responsible for the restoration of the Lorentz symmetry.

Another criterion analyzed in this work concerns the probabilistic character of processes in QFT. The unitarity of the scattering $\mathrm{S}$ matrix is a compulsory requirement to achieve any reasonable interpretation of the results of scattering processes. Since the residue of the propagator evaluated at the poles provides information on the norms of the states associated with the propagating mode, we impose that these norms must be positive definite. Considering what was said about stability and unitarity, our investigation in this work concerns the implementation of the following conditions for the absence of tachyons and ghosts:

$$
\begin{gathered}
p_{0}=f\left(\vec{p}, m^{2}\right)>0, \quad p^{2} \geq 0 \quad \forall \vec{p}, \\
\mathcal{I} \operatorname{Res}\left(\left.\Pi\right|_{p_{0}=f(\vec{p})}\right)>0,
\end{gathered}
$$

where $f\left(\vec{p}, m^{2}\right)$ is an arbitrary function of the momenta with the masses of modes, $m^{2}$, 
depending on the constants of the model, including the background vector. These functions are obtained as roots of the polynomials defined by the determinants of matrices the in Eqs. (54) - (56)

Using Eq. (29), we can rewrite the propagator as

$$
\Pi=i(-)^{R+S} \sum_{i j} S_{i}^{*} A_{i j}^{-1}\left(J M, m^{2}\right) S_{j}\left(p_{0}-f\left(\vec{p}, m^{2}\right)\right)^{-1},
$$

where $S_{i}=\psi_{i}^{a b} J_{a b}$ and $A_{i j}^{-1}\left(J M, m^{2}\right)$ is the matrix $a(J M)^{-1}$ with the pole $f\left(\vec{p}, m^{2}\right)$ extracted. Thus, the positivity condition [Eq. (58)] for arbitrary sources is guaranteed if the eigenvalues of $A_{i j}^{-1}\left(J M, m^{2}\right)$ are positive (negative) defined for the case when $R+S=0$ (1). Moreover, one can show that $A_{i j}^{-1}\left(J M, m^{2}\right)$ has only one non-null eigenvalue whenever evaluated at the pole, so that a positive (negative) eigenvalue is ensured by the positivity (negativity) of the trace $A_{i j}^{-1}\left(J M, m^{2}\right)$ evaluated at the pole. We can then rewrite the condition for the absence of ghosts as

$$
\left.(-1)^{R+S} \operatorname{tr} A_{i j}^{-1}\left(J M, m^{2}\right)\right|_{p_{0}=f\left(\vec{p}, m^{2}\right)}>0 .
$$

Taking the inverse of the matrices in Eqs. (54) - (56), we can see that each one of them presents a simple massless pole $\left(p^{2}=0\right)$ besides simple massive poles in the 14 and 23 sectors and a double massive pole in the 56 sector. The massive poles, which appear in matrices of the 14 and 23 sectors, can be put in the following general quadratic form:

$$
A_{i j} p_{0}^{2}+B_{i j} \vec{p}^{2}+C_{i j}|\vec{p}| p_{0}+D_{i j}=0
$$

where each pair of subindeces $(i, j)$ can assume the values $(1,4)$ and $(2,3)$, referring to the respective alluded sectors. These sets of parameters are given by

$$
\begin{aligned}
& A_{14}=\beta+\frac{1}{2} \kappa\left(b_{0}^{2}+\vec{b}^{2}\right) \\
& B_{14}=-\left(\beta-\frac{1}{2} \kappa\left(b_{0}^{2}-\vec{b}^{2}\right)-\kappa \vec{b}^{2} \cos ^{2} \theta\right) \\
& C_{14}=-2 \kappa b_{0}|\vec{b}| \cos \theta \\
& D_{14}=\alpha, \\
& A_{23}=\beta+\frac{1}{2} \kappa b_{0}^{2} \\
& B_{23}=-\beta+\frac{1}{2} \kappa \vec{b}^{2} \cos ^{2} \theta \\
& C_{23}=-\kappa b_{0}|\vec{p}| \cos \theta \\
& D_{23}=\alpha .
\end{aligned}
$$


For general values of the parameters, Eq. (61) may describe ellipses, parabolas, or hyperbolas on the plane $\left(p_{0},|\vec{p}|\right)$ with the center at the origin or any of its degenerate situations. For physical reasons, and in agreement with the condition for the absence of tachyons, we demand that it have two real roots for $p_{0}$ and that each one of these roots have single-valued positivity. Following our previous considerations, the positivity of energy is ensured in all reference frames if spacelike four-momenta are avoided. This can be reached by imposing the existence of asymptotes constrained to lie inside the light cone. This is only possible for the hyperbolic case. In terms of the parameters of the quadratic form, we have

$$
\begin{aligned}
C_{i j}^{2}-4 A_{i j} B_{i j} & >0, \\
\left|m_{ \pm}\right| & =\left|-\frac{C_{i j}}{2 A_{i j}} \pm \sqrt{\left(\frac{C_{i j}}{2 A_{i j}}\right)-\frac{D_{i j}}{A_{i j}}}\right| \geq 1, \\
D_{i j} / A_{i j} & <0,
\end{aligned}
$$

where $m_{ \pm}$are the slopes of the two asymptotes of the hyperbola.

The poles of the 56 sector are the solutions of the quartic equation of the form

$$
\lambda_{1} p_{0}^{4}+\lambda_{2} \vec{p}^{4}+\lambda_{3}|\vec{p}|^{3} p_{0}+\lambda_{4}|\vec{p}| p_{0}^{3}+\lambda_{5} p_{0}^{2}+\lambda_{6} \vec{p}^{2}+\lambda_{7} \vec{p}^{2} p_{0}^{2}+\lambda_{8}=0,
$$

where the coefficients $\left(\lambda_{1}, \ldots, \lambda_{8}\right)$ depend on the Lagrangian parameters and on the angle $\theta$ between the vector $\vec{b}$ and the particle momentum $\vec{p}$. In this case, there are many other possible situations for the curves in the plane $\left(p_{0},|\vec{p}|\right)$ as compared with the previous one. Obviously, there are physically reasonable possibilities among them. As a simple example, we have the interesting situation in which the quartic form is separable in the product of two quadratic ones. Within this decomposition, we can demand that each one correspond to a hyperbola with the same desired properties of the previous discussion. Due to the complexity of analyzing this dispersion relation in general, let us concentrate on the other sectors and return to this case when we discuss the unitarity of the model.

By inspecting the conditions in Eqs. (70) - (72) and using the two sets of parameters in Eqs. (62) - (65) and Eqs. (66) - (69), we conclude that the parameters of the Lagrangian 
[Eq. (1)] must satisfy the conditions

$$
\begin{aligned}
& 14 \text { Sector : } \kappa>0, \quad \beta<0 \quad b^{2}>-2\left|\frac{\beta}{\kappa}\right|, \quad\left(b_{0}^{2}+\vec{b}^{2}\right)<2\left|\frac{\beta}{\kappa}\right|, \quad \alpha>0, \\
& \kappa<0, \quad \beta>0 \quad b^{2}>-2\left|\frac{\beta}{\kappa}\right|, \quad\left(b_{0}^{2}+\vec{b}^{2}\right)<2\left|\frac{\beta}{\kappa}\right|, \quad \alpha<0, \\
& 23 \text { Sector : } \kappa>0, \quad \beta<0, \quad b_{0}^{2}<2\left|\frac{\beta}{\kappa}\right|, \quad \alpha>0, \\
& \kappa<0, \quad \beta>0, \quad b_{0}^{2}<2\left|\frac{\beta}{\kappa}\right|, \quad \alpha<0,
\end{aligned}
$$

in order to avoid instabilities coming from these sectors. It is worthwhile emphasizing that the same set of conditions is required for any choice of the background vector: $b^{2}>0, b^{2}<0$, or $b^{2}=0$.

We can assume the Sun-centered reference frame (SCRF) to be the one where the components of the background vector are sufficiently small in such a way as to match the many experiments that are in agreement with Lorentz invariance. In this sense, the relations in Eqs. (74) - (77) impose limits on the possible boosted reference frames related to the SCRF. Apparently, this dependence of the physical consistency on some restricted set of inertial observers clashes with the observer invariance of the starting Lagrangian. Nevertheless, one should be aware that our discussion about physical consistency relies on the validity of the perturbative approach to QFT and, for enough strongly boosted frames, the LV parameters are not supposed to be small anymore, jeopardizing the conclusions obtained with perturbation theory.

Let us turn to the discussion of the fulfillment of the condition in Eq. (58). As we already stated, this is needed in order to get a unitary model. For the massless pole, we can use the source constraints [Eq. (53)], the inverse of matrices (54)-(56), and the condition for the absence of ghosts [Eq. (58)] at the pole $p^{2}=0$ to get

$$
\left.J^{* a b} \frac{1}{2\left(\alpha+\kappa(v \cdot p)^{2}\right)}\left[\left(\eta_{a c} \eta_{b d}+\eta_{a d} \eta_{b c}\right)-\eta_{a b} \eta_{c d}\right]\right|_{p^{2}=0} J^{c d}>0 .
$$

We recognize the structure inside the brackets as the residue matrix of the graviton propagator. In comparison with the $\mathrm{EH}$ model, we have the appearance of the $\kappa(v \cdot p)^{2}$ term following the Newton constant $\alpha$. This contribution to the Newton constant, due to the LV term, denotes a better UV behavior, at least on the mass-shell analysis, and could have interesting consequences in the searches for renormalizable gravity models. 
To assure the positivity of the residue of the propagator for the massless mode [Eq. (78)], we must impose

$$
\left(\alpha+\kappa(v \cdot p)^{2}\right)>0 .
$$

For any type of background vector, this condition is satisfied for all $\vec{p}$ if we require that

$$
\begin{aligned}
& \kappa>0, \\
& \alpha>0 .
\end{aligned}
$$

By reanalysing the conditions for stability given in Eqs. (74) - (777), we conclude that only the conditions in Eqs. (74) and (77) are compatible with these new constraints. One important point to notice is that, even if we relax the constraint on the positivity of $\kappa$ by requiring the positivity of the combination $\left[\alpha+\kappa(v \cdot p)^{2}\right]$ up to some momentum cutoff, we still have problems with the conditions in Eqs. (75) and (77), since they require $\alpha<0$, whereas low-energy unitarity requires $\alpha>0$.

For the massive poles of the $i j$ sectors, the condition on the residues of the propagators demands that

$$
\left.\left(p_{0}-f_{i j}^{n}(\vec{p})\right) \operatorname{tra} a_{i j}^{-1}\right|_{p_{0}=f_{i j}^{n}}>0,
$$

where $f_{i j}^{n}$ means the $n$th positive energy root of the polynomial dispersion relation of the $i j$ sector. Only for the 56 sector can the $n$ index be nontrivial, since there can be two positive energy solutions for the fourth-degree polynomial [Eq. (73)]. Solving Eq. (61) with the two sets of parameters [Eqs. (62) - (69)] and imposing the conditions in Eqs. (70) - (72), we get the two positive energy solutions

$$
\begin{aligned}
& f_{14}=\frac{\kappa b_{0}(\vec{b} \cdot \vec{p})}{\beta+\frac{1}{2} \kappa\left(b_{0}^{2}+\vec{b}^{2}\right)}+\left(\left(\frac{\kappa b_{0}(\vec{b} \cdot \vec{p})}{\beta+\frac{1}{2} \kappa\left(b_{0}^{2}+\vec{b}^{2}\right)}\right)^{2}+\frac{\left(\beta-\frac{1}{2} \kappa b^{2}\right) \vec{p}^{2}-\kappa(\vec{b} \cdot \vec{p})^{2}-\alpha}{\beta+\frac{1}{2} \kappa\left(b_{0}^{2}+\vec{b}^{2}\right)}\right)^{1 / 2}, \\
& f_{23}=\frac{\kappa b_{0}(\vec{b} \cdot \vec{p})}{2\left(\beta+\frac{1}{2} \kappa b_{0}^{2}\right)}+\left(\left(\frac{\kappa b_{0}(\vec{b} \cdot \vec{p})}{2\left(\beta+\frac{1}{2} \kappa b_{0}^{2}\right)}\right)^{2}+\frac{\beta \vec{p}^{2}-\frac{1}{2} \kappa(\vec{b} \cdot \vec{p})^{2}-\alpha}{\beta+\frac{1}{2} \kappa b_{0}^{2}}\right)^{1 / 2} .
\end{aligned}
$$

Taking the inverse of the matrices (54) and (55) with the coefficients in Eqs. (47)-(51) and using the condition in Eq. (82), we get the following inequalities for the 14 and 23 sectors, respectively: 


$$
\begin{gathered}
2\left(\kappa b_{0}(\vec{b} \cdot \vec{p})\right)\left(\left(\frac{\kappa b_{0}(\vec{b} \cdot \vec{p})}{2\left(\beta+\frac{1}{2} \kappa b_{0}^{2}\right)}\right)^{2}+\frac{\beta \vec{p}^{2}-\frac{1}{2} \kappa(\vec{b} \cdot \vec{p})^{2}-\alpha}{\beta+\frac{1}{2} \kappa b_{0}^{2}}\right)^{1 / 2}> \\
-2 \frac{\left(\kappa b_{0}(\vec{b} \cdot \vec{p})\right)^{2}}{\beta+\frac{1}{2} \kappa\left(b_{0}^{2}+\vec{b}^{2}\right)}+\kappa b_{0}^{2} \vec{p}^{2}+\kappa(\vec{b} \cdot \vec{p})^{2}+\alpha, \\
\left(\kappa b_{0}(\vec{b} \cdot \vec{p})\right)\left(\left(\frac{\kappa b_{0}(\vec{b} \cdot \vec{p})}{2\left(\beta+\frac{1}{2} \kappa b_{0}^{2}\right)}\right)^{2}+\frac{\beta \vec{p}^{2}-\frac{1}{2} \kappa(\vec{b} \cdot \vec{p})^{2}-\alpha}{\beta+\frac{1}{2} \kappa b_{0}^{2}}\right)^{1 / 2}> \\
-\frac{\left(\kappa b_{0}(\vec{b} \cdot \vec{p})\right)^{2}}{2\left(\beta+\frac{1}{2} \kappa b_{0}^{2}\right)}+\frac{1}{2} \kappa(\vec{b} \cdot \vec{p})^{2}+\alpha^{2}+\frac{1}{2} \kappa b_{0}^{2} \vec{p}^{2} .
\end{gathered}
$$

From the condition in Eq. (174), we see that $\alpha>0, \beta<0, \kappa>0$, and $\beta+\frac{1}{2} \kappa\left(b_{0}^{2}+\vec{b}^{2}\right)<0$. So, the right-hand side of expression ( $(86)$ is positive definite, and so should be the left-hand side, but this is false, since $b_{0}$ and $\vec{b} \cdot \vec{p}$ have no definite sign. The same reasoning may be applied to the expression (87) with the aid of the conditions in Eq. (76)), and we again reach a contradiction.

The conclusion of this analysis is that the propagation of these modes violates the unitarity of the S matrix. The only way to try to fix this problem is inhibiting the propagation of these modes. However, if we look for the quadratic form [Eq. (61)], we note that to force the absence of these modes in any reference frame we must impose that $A=B=C=0$, and from Eqs. (62) - (69) we see that this is only possible if $\kappa=0$ and $\beta=0$. That is, the LV term in the Lagrangian [Eq. (1)] brings unavoidable inconsistencies to the quantum perturbative description of the model expanded around the flat Minkowski metric and must be switched off in order to give rise a healthy particle spectrum.

With this considerable change in the scenario, the 56 sector becomes approachable. By making $\kappa=\beta=0$, the 0 component of the spin 2 decouples from the spin 0 in the matrix [Eq. (46)], and the other coefficients become equal $(A=B=C=D)$, setting up the coefficient of the spin-2 projector. Furthermore, the expression (73) becomes

$$
\alpha \gamma p^{2}-\frac{1}{2} \alpha^{2}=0
$$

Considering our previous conditions for unitarity and stability, we must impose that $\gamma>0$ in order to get a stable spin-0 mode. The residue matrix becomes only $\frac{1}{2 \gamma p^{2}}$, and the positivity of 
$\gamma$ also ensures that this is a nonghost mode. With these choices, the model with Lagrangian $\alpha R+\gamma R^{2}$ propagates two modes: a massless spin 2, identified as the graviton, and a massive spin 0. This is a well-known model in the context of higher-derivative gravity [37]. In spite of the presence of the higher derivatives, the model must be complemented with another $\left(R_{\mu \nu} R^{\mu \nu}\right)^{2}$ term to attain renormalizability, but as we have discussed in the Introduction, this insertion spoils the unitarity.

\section{DISCUSSION AND CONCLUSIONS}

We started with a general higher-derivative gravity model added with a LV CPT-even term also containing higher derivatives, since we intended to analyze the role of the Lorentzinvariance assumption in the incompatibility of renormalizability and unitarity of higherderivative gravity models. Our guide to propose such a term was grounded on the general framework of the Standard-Model Extension, where this kind of contribution arises as a spontaneous breaking of the Lorentz symmetry at some high energy in a fundamental theory. The assumption of spontaneous instead of explicit symmetry breaking is particularly mandatory in the gravity sector of the SME if we do not want to abandon the most usual geometrical interpretation of the gravitational interaction. The most striking signal of spontaneous symmetry breaking is the appearance of extra massless and massive modes as a result of the breaking. Our position was only to discuss the metric modes by hoping that this situation would correspond to scenarios where the interaction that promotes the coupling between the metric and extra modes does not affect the quadratic Lagrangian.

To our mind, this assumption should be further investigated, since the extra modes are responsible for making the whole model consistent. An explicit example of a potential triggering the spontaneous breaking of the Lorentz symmetry and its complete analysis would shed some light on this question and also could change our conclusions. Another point to be highlighted is that, as an effective model, the SME only aims to provide lowenergy effects coming from some more fundamental theory, and as such, it cannot be pushed to extremely high energies without expecting that some inconsistencies will appear. In our investigation, we tried to discuss Lorentz violation independent of this restriction, but without clashing with it, and our general considerations of stability and unitarity point to the conclusion that the extra Lorentz noninvariant term is unable to fix the mentioned 
problem of the incompatibility of renormalizability and unitarity. Within the philosophy of effective field theories, the terms considered here can be accepted if we constrain the masses of the ghost particles to be of the same order of the cutoff energy of the model, and the new d.o.f of a more fundamental theory should be able to fix the situation. However, an effective discussion of the model should contemplate other possibilities for the violating terms that respect the same structure as the one considered here, and it would also be interesting to investigate them.

Acknowledgments

We would like to express our thanks to V.A. Kostelecký for useful discussions. C.H. wishes also to thank the International Center for Spacetime Symmetries for their kind hospitality. This work has been supported by CAPES (Coordenação de Aperfeiçoamento de Pessoal de Nível Superior-Brazil) and CNPq (Conselho Nacional de Desenvolvimento Científico e Tecnológico, Brazil).

[1] S. Weinberg, General Relativity: An Einstein Centenary Survey, edited by S. W. Hawking and W. Israel (Cambridge University Press, Cambridge, England, 1980).

[2] D. E. Neville, Phys. Rev. D 183535 (1978).

[3] D. E. Neville, Phys. Rev. D 21867 (1980).

[4] K. S. Stelle, Phys. Rev. D 16, 953 (1977).

[5] E. Sezgin and P. van Nieuwenhuizen, Phys. Rev. D 21, 3269 (1980).

[6] R. Utiyama, Phys. Rev. 101, 1597 (1956); T. W. B. Kibble, J. Math. Phys. 2, 212 (1961).

[7] V. A. Kostelecký and S. Samuel, Phys. Rev. D 39, 683 (1989).

[8] D. Colladay and V. A. Kostelecký, Phys. Rev. D 55, 6760 (1997).

[9] D. Colladay and V. A. Kostelecký, Phys. Rev. D 58, 116002 (1998).

[10] H. Belich, T. Costa-Soares, M. A. Santos, and M. T. D. Orlando, Rev. Bras. Ensino Fís. 29, 1 (2007).

[11] V. A. Kostelecký, Phys. Rev. D 69, 105009 (2004).

[12] Explicit symmetry breaking is permitted if more general geometrical frameworks are considered in the description of gravity. In this case, the geometrical entities of the manifold implement directional dependences at each spacetime point corresponding to nonzero Lorentz- 
violating coefficients. Further discussion can be found in V. A. Kostelecký, N. Russell, and R. Tso, Phys. Lett. B 716, 470 (2012).

[13] R. Bluhm, Shu-Hong Fung, and V. A. Kostelecký, Phys. Rev. D 77, 065020 (2008).

[14] R. Bluhm and V. A. Kostelecký, Phys. Rev. D 71, 065008 (2005).

[15] V. W. Hughes et al., Phys. Rev. Lett. 87, 111804 (2001); R. Bluhm, V. A. Kostelecký, and C. D. Lane, Phys. Rev. Lett. 84, 1098 (2000); E. O. Iltan, J. High Energy Phys. 06 (2003) 016.

[16] H. Nguyen (KTeV Collaboration), arXiv:hep-ex/0112046; Y. B. Hsiung et al., Nucl. Phys. B, Proc. Suppl. 86, 312 (2000); J. M. Link et al. (FOCUS Collaboration), Phys. Lett. B 556, 7 (2003); R. Ackerstaff et al. (OPAL Collaboration), Z. Phys. C 76, 401 (1997); M. Feindt et al.(DELPHI Collaboration), DELPHI report No. 97-98 CONF 80 (1997); K. Abe et al.(BELLE Collaboration), Phys. Rev. Lett. 86, 3228 (2001); B. Aubert et al.(BaBar Collaboration), arXiv:hep-ex/0303043; arXiv:hep-ex/0607103.

[17] V. A. Kostelecký and R. Potting, Phys. Rev. D 51, 3923 (1995); D. Colladay and V. A. Kostelecký, Phys. Lett. B 344, 259 (1995); Phys. Rev. D 52, 6224 (1995); V. A. Kostelecký and R. J. Van Kooten, Phys. Rev. D 54, 5585 (1996); O. Bertolami et al., Phys. Lett. B 395, 178 (1997); N. Isgur et al., Phys. Lett. B 515, 333 (2001); V. A. Kostelecký, Phys. Rev. Lett. 80, 1818 (1998); V. A. Kostelecký, Phys. Rev. D 61, 016002 (1999); V. A. Kostelecký, Phys. Rev. D 64, 076001 (2001); V. A. Kostelecký and R. J. Van Kooten, Phys. Rev. D 82, 101702(R) (2010).

[18] D. Bear, R. E. Stoner, R.L. Walsworth, V. A. Kostelecký, and C. D Lane, Phys. Rev. Lett. 85, 5038 (2000); M. A. Humphrey, D.F. Phillips, and R. L. Walsworth, Phys. Rev. A 62, 063405 (2000); D. F. Phillips, M. A. Humphrey, E. M. Mattison, R. E. Stoner, R. F. C. Vessot, and R. L. Walsworth, Phys. Rev. D 63, 111101 (2001); M. A. Humphrey et al., Phys. Rev. A 68, 063807 (2003). V. A. Kostelecký and C. D. Lane, Phys. Rev. D 60, 116010 (1999); V. A. Kostelecký and C. D. Lane, J. Math. Phys. 40, 6245 (1999); R. Bluhm, V.A. Kostelecký, C. D. Lane, and N. Russell, Phys. Rev. Lett. 88, 090801 (2002); F. Canè et al., Phys. Rev. Lett. 93, 230801 (2004); P. Wolf, F. Chapelet, S. Bize, and A. Clairon, Phys. Rev. Lett. 96, 060801 (2006).

[19] S. M. Carroll, G. B. Field, and R. Jackiw, Phys. Rev. D 41, 1231 (1990); V. A. Kostelecký and M. Mewes, Phys. Rev. Lett. 87, 251304 (2001); J. A. Lipa, J. A. Nissen, S. Wang, D. A. Stricker, and D. Avaloff, Phys. Rev. Lett. 90, 060403 (2003); Q. G. Bailey and V. A. 
Kostelecký, Phys. Rev. D 70, 076006 (2004); R. Lehnert and R. Potting, Phys. Rev. Lett. 93, 110402 (2004); Phys. Rev. D 70, 125010 (2004); B. Feng, M. Li, J.-Q. Xia, X. Chen, and X. Zhang, Phys. Rev. Lett. 96, 221302 (2006); V. A. Kostelecký and M. Mewes, Phys. Rev. Lett., Phys. Rev. Lett. 97, 140401 (2006); B. Altschul, Phys.Rev.Lett. 98, 041603 (2007); V. A. Kostelecký and M. Mewes, Astrophys. J. Lett. 689, L1 (2008); Phys. Rev. D 80, 015020 (2009); Phys. Rev. Lett. 110, 201601 (2013).

[20] H. Dehmelt, R. Mittleman, R. S. Van Dyck, Jr., and P. Schwinberg, Phys. Rev. Lett. 83, 4694 (1999); R. K. Mittleman, I. I. Ioannou, H. G. Dehmelt, and N. Russell, Phys. Rev. Lett. 83, 2116 (1999); G. Gabrielse et al., Phys. Rev. Lett. 82, 3198 (1999); R. Bluhm, V. A. Kostelecký, and N. Russell, Phys. Rev. Lett. 82, 2254 (1999); Phys. Rev. Lett. 79, 1432 (1997); R. Bluhm, V. A. Kostelecký and N. Russell, Phys. Rev. D 57, 3932 (1998); C. D. Lane, Phys. Rev. D 72, 016005 (2005); L.-S. Hou, W.-T. Ni, and Y.-C.M. Li, Phys. Rev. Lett. 90, 201101 (2003); R. Bluhm and V. A. Kostelecký, Phys. Rev. Lett. 84, 1381 (2000); B. R. Heckel et al., Phys. Rev. Lett. 97, 021603 (2006); H. Müller, S. Herrmann, A. Saenz, A. Peters, and C. Lämmerzahl, Phys. Rev. D 68, 116006 (2003); R. Lehnert, J. Math. Phys. 45, 3399 (2004); B. Altschul, Phys. Rev. Lett. 96, 201101 (2006).

[21] V. Barger, S. Pakvasa, T. J. Weiler, and K. Whisnant, Phys. Rev. Lett. 85, 5055 (2000); J. N. Bahcall et al., Phys. Lett. B 534, 114 (2002); V. A. Kostelecký and M. Mewes, Phys. Rev. D 70, 031902 (2004); V. A. Kostelecký and M. Mewes, Phys. Rev. D 70, 076002 (2004); T. Katori, V. A. Kostelecký, and R. Tayloe, Phys. Rev. D 74, 105009 (2006); V. A. Kostelecký and M. Mewes, Phys. Rev. D 69, 016005 (2004). J. S. Díaz, V. A. Kostelecký, and M. Mewes, Phys. Rev. D 80, 076007 (2009); V. A. Kostelecký and M. Mewes, Phys. Rev. D 85, 096005 (2012); J. S. Díaz and V. A. Kostelecký, Phys. Rev. D 85, 016013 (2012); J. S. Díaz, V. A. Kostelecký, and R. Lehnert, Phys. Rev. D 88, 071902(R) (2013); J. S. Díaz, V. A. Kostelecký, and M. Mewes, Phys. Rev. D 89, 043005 (2014).

[22] D. L. Anderson, M. Sher, and I. Turan, Phys. Rev. D 70, 016001 (2004).

[23] Q. G. Bailey and V. A. Kostelecký, Phys. Rev. D 74, 045001 (2006).

[24] V. A. Kostelecký, N. Russell, and J. D. Tasson, Phys. Rev. Lett. 100, 111102 (2008).

[25] V. A. Kostelecký and J. D. Tasson, Phys. Rev. Lett. 102, 010402 (2009).

[26] V. A. Kostelecký and J. D. Tasson, Phys. Rev. Lett. 102, 010402 (2009).

[27] V. A. Kostelecký and N. Russell, Rev. Mod. Phys. 83, 11 (2011). 
[28] The discussion of the stability and unitarity of a Lorentz-violating gravity model without higher derivatives, but taking into account the extra modes coming from the spontaneous symmetry-breaking mechanism was performed in R. V. Maluf, C. A. S. Almeida, R. Casana, and M. M. Ferreira, Jr. arXiv:1402.3554.

[29] B. Pereira-Dias, C. A. Hernaski, and J. A. Helayël-Neto, Phys. Rev. D 83, 084011 (2011).

[30] S. Weinberg, Phys. Rev. 138, B988 (1965).

[31] R. J. Rivers, Il Nuovo Cimento 34, 387 (1964).

[32] C. W. Misner, K. S. Thorne, and J. A. Wheeler, Gravitation (W. H. Freeman and Company, San Francisco, 1973).

[33] V. A. Kostelecký and R. Lehnert, Phys. Rev. D 63, 065008 (2001).

[34] C. Adam and F. R. Klinkhamer, Nucl. Phys. B607, 247 (2001).

[35] C. M. Reyes, Phys. Rev. D 82, 125036 (2010).

[36] R. Bluhm, N. L. Gagne, R. Potting, and A.Vrublevskis, Phys.Rev. D 77, 125007 (2008).

[37] P. van Nieuwenhuizen, Nucl. Phys. B60, 478 (1973). 